\title{
On the stress overshoot in cluster crystals under shear
}

\author{
G.P. Shrivastav, G. Kahl \\ Institut für Theoretische Physik and Center for Computational Materials Science (CMS), TU Wien, \\ Wiedner Hauptstraße 8-10, A-1040 Wien, Austria
}

Received January 31, 2020, in final form March 3, 2020

\begin{abstract}
Using non-equilibrium molecular dynamics simulations we study the yielding behaviour of a model cluster crystal formed by ultrasoft particles under shear. We investigate the evolution of stress as a function of strain for different shear rates, $\dot{\gamma}$, and temperatures. The stress-strain relation displays a pronounced maximum at the yielding point; the height of this maximum, $\sigma_{\mathrm{p}}$, increases via a power law with an increasing shear range and tends to saturate to a finite value if the limit shear rate goes to zero (at least within the considered temperature range). Interestingly, this behaviour can be captured by the Herschel-Bulkley type model which, for a given temperature, allows us to predict a static yield stress $\sigma_{\mathrm{p}}^{0}$ (in the shear rate tending to zero limit), a characteristic timescale $\tau_{\mathrm{c}}$, and the exponent $\alpha$ of the above-mentioned power-law decay of the $\sigma_{\mathrm{p}}$ at high shear rates. Furthermore, for different temperatures, the $\sigma_{\mathrm{p}}$ can be scaled as functions of $\dot{\gamma}$ onto a single master curve when scaled by corresponding $\tau_{\mathrm{c}}$ and $\sigma_{\mathrm{p}}^{0}$. Moreover, for a given shear rate, $\sigma_{\mathrm{p}}$ displays a logarithmic dependence on temperature. Again, the $\sigma_{\mathfrak{p}}-T$ curves for different shear rates can be scaled on a single logarithmic master curve when scaled by a corresponding fitting parameters.
\end{abstract}

Key words: rheology, cluster crystals, yielding, molecular dynamics, stress overshoot

\section{Introduction}

Cluster crystals [1] represent an archetypical ordered phase encountered in a particular class of soft matter systems, so-called ultrasoft particles, whose interaction at vanishing interparticle distance attains a finite energy penalty: such cluster phases can be found in systems if the Fourier transform of the potentials shows negative components [2]. At low and intermediate densities, a disordered phase of clusters of overlapping particles is found, characterized by a rather polydisperse distribution in the cluster occupancy. If at a fixed temperature the density is increased this disordered phase transforms via a first order phase transition into a cluster BCC phase and then - upon further increasing the density - into a cluster FCC phase. In these ordered particle configurations, the lattice sites of the BCC and of the FCC lattice are occupied by clusters of overlapping particles which are now rather monodisperse in their cluster occupancy [1, 3--7]. Even though the fact that mutually repelling particles are capable of forming stable clusters is at least at first glance counter-intuitive, the physics behind this intriguing phase is meanwhile well understood in terms of classical density functional theory [8, 9]. Furthermore, in the two-dimensional case, the effect of particle-size on the phase diagram of cluster crystals has been explored [10]. The size of particles is incorporated by introducing a hardcore repulsion in the interaction potential [10, 11]. In contrast to the fluid cluster-crystal phases where particles inside clusters remain disordered, crystal cluster-crystals are obtained at low temperatures which are characterized by the ordered structure of particles inside a cluster [10].

Later contributions in literature have been dedicated to the equilibrium dynamics of cluster crystals [7, [12]. Again, these systems show an intriguingly new behaviour: the longtime dynamics is diffusive which is the result of the particles hopping from one cluster to a neighboring cluster, the distribution of the jump length is found to be exponential at short distances. At large distances, this distribution follows a 
power-law decay, i.e., a behaviour reminiscent of Lévy flights [13]. As expected, the diffusion coefficient exhibits the Arrhenius behaviour confirming that the hoping of particles is an activated process. This particular type of diffusion of particles makes cluster crystals an appropriate model system to study the mechanical response of defect-rich crystals [14], notably the effect of the defect-dynamics on the yielding of crystalline solids, an area which is less explored in the recently developed theories to understand the deformation of crystalline solids [15, 16].

Just recently, out-of-equilibrium investigations have been dedicated to cluster crystals [12, 17, 18]. In a recent computer simulation study on the steady-state rheology of cluster crystals, an intriguing shear-induced self-assembly was observed, namely a shear-induced fluidization (i.e., string formation) at very high shear rates. When the shear rate is further decreased, string-like structures appear and at very low shear rates a shear shear-banding regime occurs. The shear viscosity for cluster crystals can be obtained in the steady state via the ratio of the shear stress and the shear rate. In fact, in a previous computer simulation by Nikoubashman et al. [17] on GEM-8 cluster crystals, it was shown that at a given temperature, the shear viscosity decreases in a power-law manner with an increasing shear rate. In this work, we are focusing on the yielding behaviour of cluster crystals. Therefore, we do not consider the steady state scenario. However, we expect a qualitatively similar response for a GEM-4 cluster crystal (which is the focus of the present study).

Furthermore, the dependence of the shear viscosity on the temperature (for a given shear rate) has not been studied systematically in literature so far. Considering the previously mentioned study by Coslovich et al. [13] on the equilibrium diffusion of particles in cluster crystals, we expect that the shear viscosity should decrease with an increasing temperature. In an effort to understand the effect of temperature on the shear viscosity of cluster crystals, a further detailed study is needed.

This contribution is dedicated to the yielding behaviour of cluster crystals, a feature which, so far, has not been investigated at all. In particular, we investigate the effect of shear rate and temperature on the mechanical failure of these materials. In this work, we study with the help of extensive computer simulations the yielding behaviour of the cluster crystals under steady shear conditions by examining the response of the stress as a function of strain. We will show that the stress exhibits a maximum after an initial linear increase; the height of this maximum depends on the shear rate and temperature. Similar to the majority of the preceding investigations on cluster forming systems, our investigations are based on the so-called generalized exponential model interaction with the index $n$ (GEM- $n$ ), a potential which combines the bounded nature of the interaction (required to ensure clustering) with a functionally simple mathematical form that is easily amenable to computer simulations. We will show that the stress exhibits a pronounced maximum after the initial, linear increase. The height of this maximum depends on the shear rate and on the temperature; in this contribution we analyse these dependencies via suitably chosen laws.

The paper is organized as follows: in section 2, we introduce the model and details of the simulations and the related protocols. Results are presented and discussed in section 3 , while the final section contains concluding and summarizing remarks and an outlook to future, related investigations.

\section{Model and simulation details}

In our cluster crystal system, particles interact via the so-called generalized exponential (GEM- $n$ ) potential [1], assuming in this contribution $n=4$; this interaction is defined via

$$
\Phi(r)=\epsilon \exp \left[-(r / d)^{4}\right],
$$

where $d$ and $\epsilon$ set the length- and energy-scales of the model, respectively; note that we use - in contrast to the usual notation in the previous, related contributions - the symbol $d$ for the range of the interparticle interaction, since the conventional symbol $\sigma$ is reserved in this manuscript to denote the stress.

In the simulations, the GEM-4 potential is truncated and shifted to zero at a distance $r_{\mathrm{c}}=2.2 d$. The units of temperature $(T)$, density $(\rho)$, and time $(t)$, are given by $k_{\mathrm{B}} T / \epsilon, \rho d^{3}$, and $t_{0}=d \sqrt{m / \epsilon}$, respectively. Here, $m$ is the mass of particles and $k_{\mathrm{B}}$ is Boltzmann's constant. In our simulations we set the values of $\epsilon, d, m$, and $k_{\mathrm{B}}$ equal to unity. 


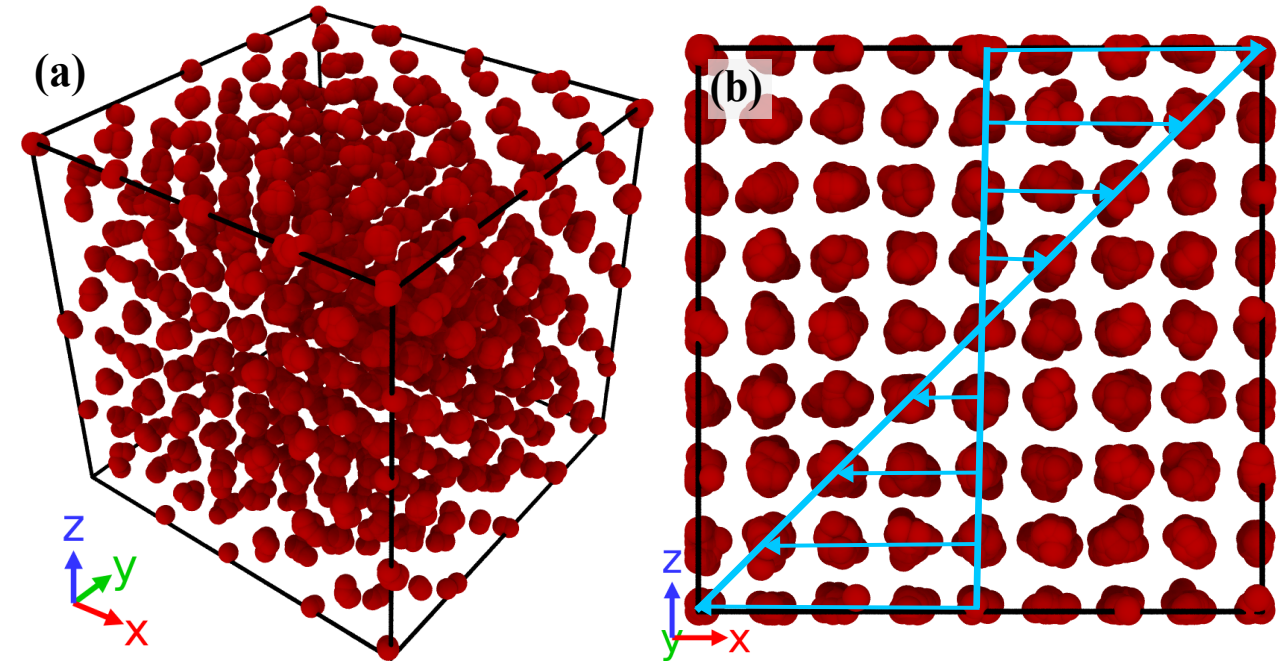

Figure 1. (Colour online) Panel (a): snapshot of an equilibrium configuration cluster crystal in the FCC phase at $\rho=6.5$ and $T=0.6$ (see text); this snapshot was created using the graphic package OVITO [22]. Panel (b): a schematic view of the simulation cell which illustrates how the shear flow is imposed in the $(x, z)$-plane on the FCC cluster crystal [using the snapshot shown in panel (a)] along the shear direction $x$. The blue arrows visualize the velocity gradient along the $z$-direction.

All investigations were carried out with the LAMMPS simulation package [19]: we performed molecular dynamics (MD) simulations in an NVT ensemble of $N=3328$ particles (with periodic boundary conditions), integrating Newton's equations-of-motions with the Verlet velocity integration scheme [20]; a time increment of $\Delta t=0.005 t_{0}$ was used. The temperature was kept constant with the help of a DPD thermostat which preserves the hydrodynamics [21]. For each state point, 50 independent simulation runs were performed; observables were then obtained by averaging the related data over these runs.

The system was studied at a fixed density, namely $\rho=6.5$, and at five different temperatures, i.e., $T=0.8,0.75,0.7,0.65$, and 0.6. At these state points, the system is in a stable FCC cluster phase, where each site of the FCC lattice is occupied by a cluster of overlapping particles (see phase diagram shown in [1]). According to the results available in literature [1, 5, 13] the average number of particles $N_{\mathrm{c}}$ pertaining to cluster ranges for the considered state points around the value $N_{\mathrm{c}} \simeq 13$.

The initial configurations for our simulations are ideal FCC cluster crystals where each lattice site is occupied by $N_{\mathrm{c}}=13$ completely overlapping particles and assuming a lattice distance that is compatible with the given value of $\rho$. Starting from this configuration, the system is equilibrated independently at each of the above specified temperature values over $10^{6} \mathrm{MD}$ steps. A snapshot of the resulting equilibrium configuration (at $\rho=6.5$ and $T=0.6$ ) is shown in panel (a) of figure 1 .

We shear the bulk cluster crystal by imposing a planar Couette flow in the $(x, z)$-plane and use LeesEdwards boundary conditions [23]. To this end, a constant shear rate is applied along the $x$-direction; thus, in this setup the $z$ - and $y$-directions are the gradient- and vorticity-directions, respectively. The shear rate, $\dot{\gamma}$, considered in our investigations ranges from, $\dot{\gamma}=10^{-5}$ to $\dot{\gamma}=10^{-1}$ (given in units of $t_{0}^{-1}$ ). A schematic illustration of the setup of our shearing experiment is presented in panel (b) of figure1; here, the front view of the snapshot (i.e., its projection onto the $(x, z)$-plane) of the equilibrated cluster crystal (as displayed in panel (a) of figure 1 is shown; the velocity gradient is indicated by the blue arrows.

We note that in contrast to [17], we consider here rather low shear rates for the following reasons: (i) the DPD thermostat (as implemented in the LAMMPS package) becomes in our investigations unstable at shear rates larger than $\dot{\gamma}=10^{-1}$; (ii) the present work focuses on an understanding of the yielding behaviour of cluster crystals in the limit of vanishing shear rate; still it should be mentioned that, on the other hand, also very low shear rates (i.e., $\dot{\gamma} \lesssim 10^{-5}$ ) are difficult to access, because the related simulations become computationally expensive. 


\section{Results}

In an effort to understand the yielding of the cluster crystal under shear, we record the evolution of the stress, $\sigma_{x z}(t)$, during the shearing experiment as a function of strain, $\dot{\gamma} t$, assuming different values for the shear rate $\dot{\gamma}$.

From the quantities available during the simulation run, the stress $\sigma_{x z}(t)$ is calculated via the IrvingKirkwood expression [24]:

$$
\left\langle\sigma_{x z}(t)\right\rangle=\frac{1}{V}\left\langle\sum_{i}\left[m v_{i, x}(t) v_{i, z}(t)+\sum_{i>j} r_{i j, x}(t) F_{i j, z}(t)\right]\right\rangle .
$$

Bearing in mind that $m$ and $d$ are set to unity, the unit of $\left\langle\sigma_{x z}(t)\right\rangle$ is $1 / t_{0}^{2}$. In the above relation, $V$ represents the total volume of the system, $m$ is the mass of the particles, $v_{i, x}(t)$ and $v_{i, z}(t)$ represent the $x$ - and $z$-components of the velocity of particle $i, r_{i j, x}(t)$ is the $x$-component of the displacement vector between particles $i$ and $j$, and $F_{i j, z}(t)$ denotes the $z$-component of the force between particles $i$ and $j$. The angular brackets in relation (3.1) represent an averaging over the aforementioned 50 independent runs.

In the following subsections we discuss the impact of the shear rate and of the temperature on the stress.

\subsection{Impact of the shear rate on the stress}

In panels (a) and (b) of figure 2 we display the time-evolution of the stress, $\left\langle\sigma_{x z}(t)\right\rangle$, as a function of the strain $\dot{\gamma} t$ for five different shear rates, namely $\dot{\gamma}=10^{-1}, 10^{-2}, 10^{-3}, 10^{-4}$, and $10^{-5}$ at temperatures $T=0.6$ [panel (a)] and $T=0.8$ [panel (b)]; similar graphs also exist for the other temperatures investigated, but they are not on display here. For all values of $\dot{\gamma}$ we observe that the stress first increases with the shear rate and then reaches a pronounced maximum. At this peak in the stress-strain response curve the cluster crystal yields and the stress decays beyond this maximum. We observe that this decay becomes sharper (eventually even abrupt) as the shear rate is decreased. Beyond this decay, essentially two different archetypical scenarios of the stress-strain curve can be identified: (i) for moderate shear rates (i.e., for $\dot{\gamma} \simeq 10^{-1}$ ) the curve levels off without any significant characteristic features; (ii) for smaller shear rates, however, the stress-shear curves show characteristic secondary peaks, which become more and more pronounced as the shear rates decrease. These additional peaks can be attributed to partial release events of the stress via local particle arrangements, a feature which becomes more pronounced as the shear rate is lowered. When after such a local rearrangement event the strain increases, the cluster crystal yields again and another secondary peak appears in the stress-strain curve which has a lower height than the preceding (and the primary) peak.

The data presented in panels (a) and (b) of figure 2 also provide evidence that at a fixed temperature, the height of the above mentioned peak in the stress, denoted henceforward as $\sigma_{\mathrm{p}}$, decreases as the shear rate is lowered. This also indicates that the resistance applied by the cluster crystal to the shear forces reduces as the shear rate is lowered. This observation is visualized in panel (c) of figure 2, where we have plotted $\sigma_{\mathrm{p}}$ as a function of the shear rate $\dot{\gamma}$ for all the temperatures investigated. In an effort to quantify this effect and to provide more insight into the observed phenomena, we have fitted $\sigma_{\mathrm{p}}(\dot{\gamma})$ via the following functional form, known in literature as the Herschel-Bulkley type expression [25, 26], which was originally developed for the steady state, but which we extend now to the out-of-equilibrium case:

$$
\sigma_{\mathrm{p}}(T, \dot{\gamma})=\sigma_{\mathrm{p}}^{0}(T)+A(T) \dot{\gamma}^{\alpha}
$$

The maximum in the stress-strain curve $\sigma_{\mathrm{p}}$ is also referred to in literature as the "static yield stress" [26]. In case of amorphous solids, it was observed in computer simulations that the stress overshoot shows a logarithmic dependence on the shear rate [27, 28]. By fitting the simulation data to the above expression one can extract a value for $\sigma_{\mathrm{p}}^{0}$, i.e., the value of the static yield stress as $\dot{\gamma}$ tends to zero; we conclude that $\sigma_{\mathrm{p}}^{0}$ will correspond to the yield stress which is defined as the minimum stress required to initiate a plastic deformation in the cluster crystal [26]. We note that alternatively, this quantity could also be calculated from constant stress simulations [27], an issue which we postpone to future investigations. 

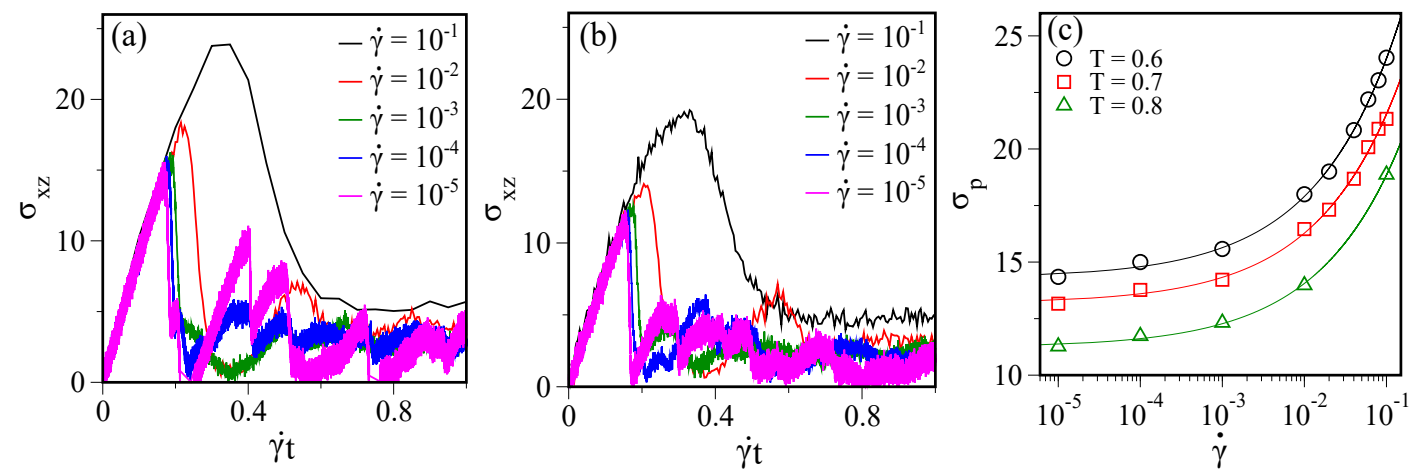

Figure 2. (Colour online) Panels (a) and (b): time evolution of the stress, $\left\langle\sigma_{x z}(t)\right\rangle$, as a function of strain, $\dot{\gamma} t$, for different shear rates, $\dot{\gamma}=10^{-1}, 10^{-2}, 10^{-3}, 10^{-4}$ and $10^{-5}$ (as labeled), for the case $T=0.6$ and 0.8 , respectively. Panel (c): variation of the stress overshoot, $\sigma_{\mathrm{p}}$ (as defined and discussed in the text), as a function of the shear rate, $\dot{\gamma}$, for different temperatures (as labeled) in a semi-logarithmic plot. In this panel, the solid lines are given by the Herschel-Bulkley type fitting function defined in equation (3.2).

Table 1. Parameters $\sigma_{\mathrm{p}}^{0}$ and $A(T)$ as obtained by fitting the simulation data for $\sigma_{\mathrm{p}}$ via the Herschel-Bulkley type expression, given in equation (3.2) for the five different temperatures investigated in this contribution. The temperature-dependent timescale $\tau_{\mathrm{c}}$ is calculated from $A$ and $\sigma_{\mathrm{p}}$ (as specified in the table).

\begin{tabular}{|c||c|c|c|}
\hline$T$ & $\sigma_{\mathrm{p}}^{0}$ & $A$ & $\tau_{\mathrm{c}}=\left(A / \sigma_{\mathrm{p}}^{0}\right)^{1 / \alpha}$ \\
\hline 0.60 & 14.299 & 26.110 & 4.056 \\
\hline 0.65 & 13.656 & 24.132 & 3.759 \\
\hline 0.70 & 13.170 & 22.526 & 3.484 \\
\hline 0.75 & 12.034 & 22.373 & 4.230 \\
\hline 0.80 & 11.224 & 20.517 & 4.067 \\
\hline
\end{tabular}

The temperature-dependent parameter $A(T)$ in the above equation is related to a characteristic timescale, $\tau_{\mathrm{c}}(T)$, which we introduce below in equation 3.3 . Finally, $\alpha$ is the exponent that characterizes the power-law decay of the stress overshoot as a function of $\dot{\gamma}$.

The solid lines in panel (c) of figure 2 show the simulation data, along with the curves which emerge by fitting these results by the Herschel-Bulkley type expression defined in equation (3.2). The emerging values of $\sigma_{\mathrm{p}}^{0}(T)$ and $A(T)$ are listed in table 1 . The exponent $\alpha$ is found to be essentially independent of the temperature in the considered range of temperatures, namely $\alpha \simeq 0.43$. It is worth mentioning that a related exponent $\alpha$ occurs also in the Herschel-Bulkley model for the flow curve (i.e., the variation of the steady-state stress as a function of shear rate). We leave investigations related to this issue to later contributions.

The static yield stress, $\sigma_{\mathrm{p}}^{0}(T)$, is displayed as a function of temperature in panel (a) of figure 3 . This quantity shows an essentially linear decay with an increasing temperature, a feature that can probably be attributed to the fact that for a cluster crystal at equilibrium the number of particles that hop from one cluster to the neighbouring one decreases with a decreasing temperature.

Further, the different curves $\sigma_{\mathrm{p}}=\sigma_{\mathrm{p}}(\dot{\gamma})$, as shown in panel (c) of figure 2 can be mapped onto one single master curve by scaling the shear rate by a temperature-dependent timescale, $\tau_{\mathrm{c}}(T)$, the latter one being defined as $\tau_{\mathrm{c}}=\left(A / \sigma_{\mathrm{p}}^{0}\right)^{1 / \alpha}$; the values of this timescale for different temperatures are accumulated in table 1. Starting from equation (3.2) this master curve has the following form [29]:

$$
\frac{\sigma_{\mathrm{p}}(\dot{\gamma})}{\sigma_{\mathrm{p}}^{0}}=1+\left(\tau_{\mathrm{c}} \dot{\gamma}\right)^{\alpha} .
$$

In panel (b) of figure 3 we show this master curve onto which the different $\sigma_{\mathrm{p}}(\dot{\gamma})$-curves can be mapped. 

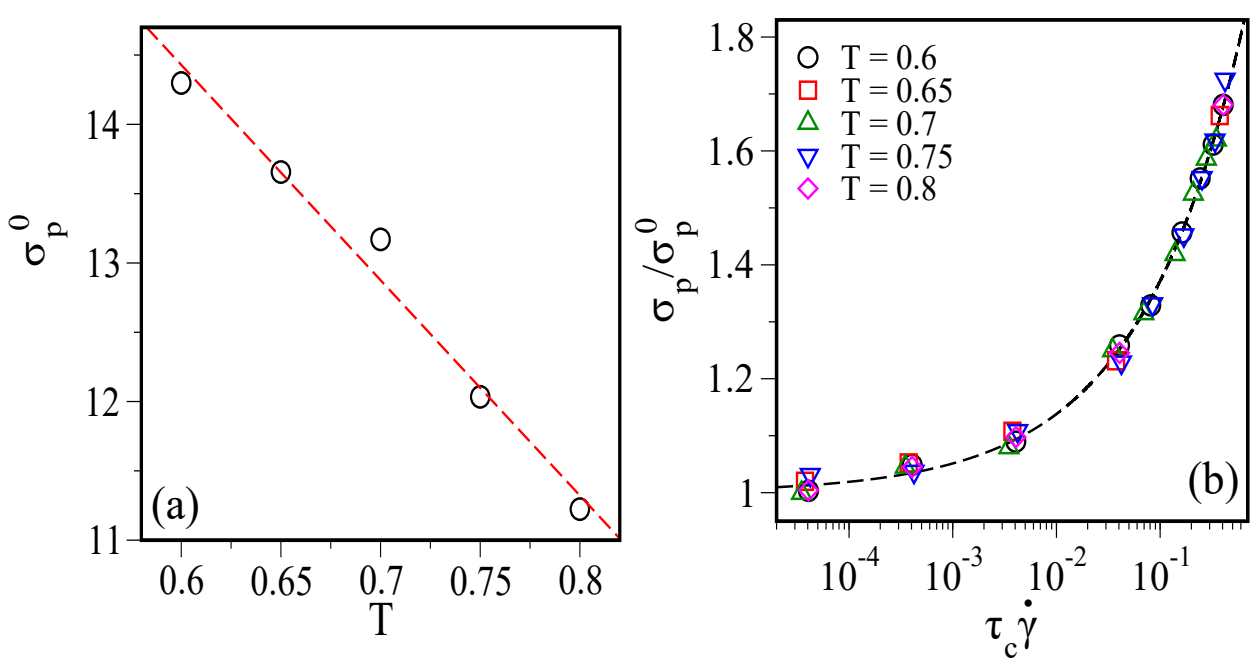

Figure 3. (Colour online) Panel (a): static yield stress, $\sigma_{\mathrm{p}}^{0}(T)$, as a function of temperature $T$, as obtained by fitting the data of $\sigma_{\mathrm{p}}(\dot{\gamma})$ shown in panel (c) of figure 2 to the Herschel-Bulkley type expression, equation 3.2. Panel (b): the symbols show the results of $\sigma_{\mathrm{p}} / \sigma_{\mathrm{p}}^{0}$ (i.e., the data shown in panel (b) of figure 2 as a function of the shear rate, $\dot{\gamma}$, now scaled by the temperature-dependent timescale $\tau_{\mathrm{c}}$ (see text) for different temperatures (as labeled). The black dotted line represents the functional form of $\sigma_{\mathrm{p}}(\dot{\gamma}) / \sigma_{\mathrm{p}}^{0}$, as given in equation 3.3 .

Such a scaling behaviour provides evidence of a universal scenario of yielding in cluster crystals which — at least in the considered range of temperatures — is independent of the temperature.

\subsection{The effect of temperature}

It can be seen from panel (c) of figure 2 that the yielding of cluster crystals strongly depends on the temperature. In the following, we analyze this dependence by investigating the behaviour of $\sigma_{\mathrm{p}}$ as a function of temperature for different shear rates. Data shown in panel (a) of figure 4 provide evidence that $\sigma_{\mathrm{p}}$ decreases logarithmically as the temperature increases for all the shear rates considered. Thus, the data can be fitted via

$$
\sigma_{\mathrm{p}}(T, \dot{\gamma})=B(\dot{\gamma})-C(\dot{\gamma}) \ln T,
$$

where $B(\dot{\gamma})$ and $C(\dot{\gamma})$ are suitably chosen parameters. The dashed lines in panel (a) of figure 4 represent the fitting function defined in the above equation. The related coefficients, $B(\dot{\gamma})$ and $C(\dot{\gamma})$, shown in figure 4 (b), are themselves functions of the shear rate: (i) $B(\dot{\gamma})$ decreases at high shear rates with $\dot{\gamma}$, it saturates at low shear rates; (ii) the $\dot{\gamma}$-dependence of $C(\dot{\gamma})$ does not show any significant features. Finally, we point out that the quantity $\left[B(\dot{\gamma})-\sigma_{\mathrm{p}}(T)\right] / D(\dot{\gamma})=\ln T$ can be mapped onto a single master curve, shown in panel (c) of figure 4 as a black dashed line.

It should be noted that such a logarithmic decay of $\sigma_{\mathrm{p}}$ as a function of temperature is also observed in thermal glasses [27, 28, 30]. This is the more astonishing since such systems are metastable, thus the age of the sample becomes an important feature. Cluster crystals, on the other hand, are equilibrium systems, and aging is in this case not a relevant phenomenon.

\section{Conclusion and outlook}

For this contribution we have made an extensive simulation-based study to understand the impact of the shear rate and of the temperature on the yielding of cluster crystals which is exposed to shear forces. In particular, we investigated the behaviour of the stress overshoot for different shear rates and temperatures 

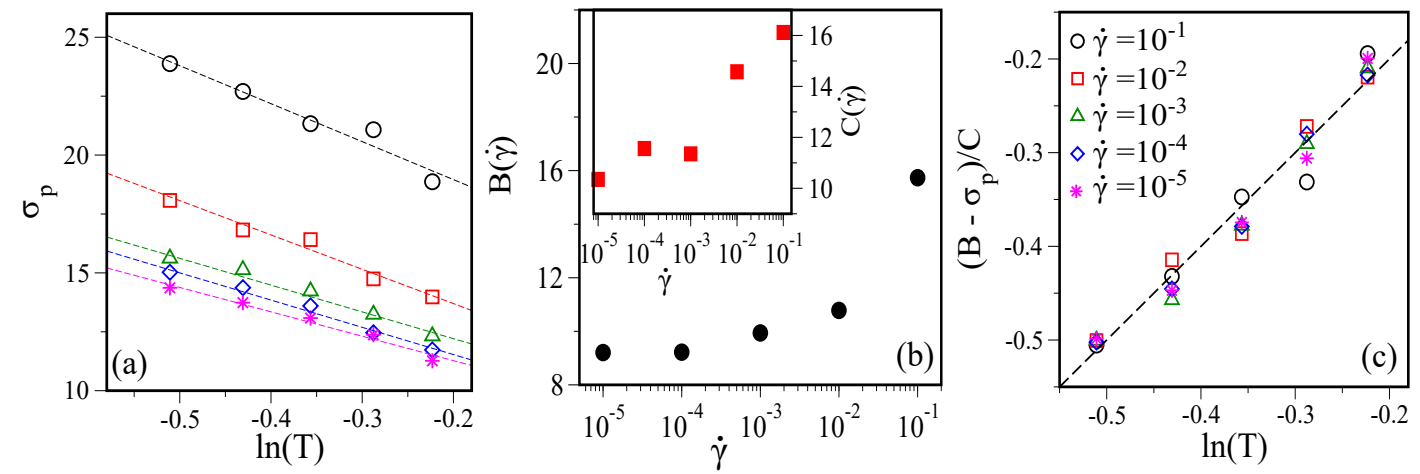

Figure 4. (Colour online) Panel (a): variation of the stress overshoot, $\sigma_{\mathrm{p}}$, as a function of temperature (in logarithmic units) for shear rates, $\dot{\gamma}=10^{-1}, 10^{-2}, 10^{-3}, 10^{-4}$ and $10^{-5}$ [as labeled in panel (c)]. The solid lines originate from the logarithimic fittig function, defined in equation (3.4). Panel (b): variation of the fitting parameters $B(\dot{\gamma})$ and $C(\dot{\gamma})$ (see the inset), introduced in equation (3.4), as functions of the shear rate. Panel (c): Scaling of $\left(B-\sigma_{\mathrm{p}}\right) / C$ (shown as a dashed black line) as a function of the temperatures (on a logarithmic scale). The symbols are the related simulation data.

by analysing the stress-strain curve. We have found that for a given temperature the height of the stress overshoot, $\sigma_{\mathrm{p}}$, increases in a power-law manner with an increasing shear rate in a Herschel-Bulkley type manner; the related exponent is essentially independent of temperature - at least for the range of the temperatures considered in this work. At low shear rates, $\sigma_{\mathrm{p}}^{0}$ tend to saturate to a finite value, $\sigma_{\mathrm{p}}^{0}$. However, we note here that a systematic finite-size analysis is needed to ensure the non-vanishing value of $\sigma_{\mathrm{p}}^{0}$. Further, the behaviour of the stress overshoot as a function of shear rate is found to have a universal nature, thus different curves of $\sigma_{\mathrm{p}}(\dot{\gamma})$ can be mapped onto a single master curve. The static yield stress is found to decay in a linear manner with temperature. At a given shear rate, the height of the stress overshoot exhibits a logarithmic dependence on the temperature. This behaviour is also found to be universal, thus different $\sigma_{\mathrm{p}}(T)$ curves can be mapped onto a single master curve. We note that this logarithmic behaviour is a characteristic feature of defect-rich systems such as thermal glasses [27, 28], as also predicted by the Ree-Eyring viscosity theory [25, 31].

Further, a systematic understanding of the mechanical response of cluster crystals is still missing. A complete calculation of the various elastic constants would be desirable but it requires expensive computer simulations. The bulk modulus of cluster crystals, studied via density functional theory and Monte Carlo simulations was discussed by Mladek et al. [32]. In later years, these results for the bulk modulus were compared with the related data obtained from the dispersion relations for cluster crystals by Häring et al. [14].

\section{Acknowledgements}

The authors acknowledge financial support by the Austrian Science Foundation (FWF) under Proj. No. I3846. The computational results presented have been achieved using the Vienna Scientific Cluster (VSC). GK would like to express his gratitude towards Ihor Mryglod for so many years of strong personal and scientific ties.

\section{References}

1. Mladek B.M., Gottwald D., Kahl G., Neumann M., Likos C.N., Phys. Rev. Lett., 2006, 96, No. 4, 045701, doi $10.1103 /$ PhysRevLett.96.045701

2. Likos C., Lang A., Watzlawek M., Löwen H., Phys. Rev. E, 2001, 63, No. 3, 031206, doi 10.1103/PhysRevE.63.031206. 
3. Zhang K., Charbonneau P., Mladek B.M., Phys. Rev. Lett., 2010, 105, No. 24, 245701, doi 10.1103/PhysRevLett.105.245701

4. Wilding N.B., Sollich P., EPL, 2013, 101, No. 1, 10004, doi 10.1209/0295-5075/101/10004

5. Mladek B.M., Gottwald D., Kahl G., Neumann M., Likos C.N., J. Phys. Chem. B, 2007, 111, No. 44, 1279912808, doi:10.1021/jp074652m

6. Likos C.N., Mladek B.M., Moreno A.J., Gottwald D., Kahl G., Comput. Phys. Commun., 2008, 179, No. 1-3, 71-76, doi:10.1016/j.cpc.2008.01.015

7. Mladek B.M., Charbonneau P., Likos C.N., Frenkel D., Kahl G., J. Phys.: Condens. Matter, 2008, 20, No. 49, 494245, doi $10.1088 / 0953-8984 / 20 / 49 / 494245$

8. Likos C.N., Mladek B.M., Gottwald D., Kahl G., J. Chem. Phys., 2007, 126, No. 22, 224502, doi $10.1063 / 1.2738064$

9. Evans R., Oettel M., Roth R., Kahl G., J. Phys.: Condens. Matter, 2016, 28, No. 24, 240401, doi $10.1088 / 0953-8984 / 28 / 24 / 240401$

10. Caprini L., Hernández-García E., López C., Phys. Rev. E, 2018, 98, No. 5, 052607, doi $10.1103 /$ physreve.98.052607

11. Delfau J.B., Ollivier H., López C., Blasius B., Hernández-García E., Phys. Rev. E, 2016, 94, No. 4, 042120, doi $10.1103 /$ physreve.94.042120

12. Nikoubashman A., Kahl G., Likos C.N., Soft Matter, 2012, 8, No. 15, 4121-4131, doi 10.1039/C1SM06899G

13. Coslovich D., Strauss L., Kahl G., Soft Matter, 2011, 7, No. 5, 2127-2137, doi 10.1039/C0SM00545B

14. Häring J.M., Walz C., Szamel G., Fuchs M., Phys. Rev. B, 2015, 92, No. 18, 184103, doi 10.1103/PhysRevB.92.184103.

15. Reddy V.S., Nath P., Horbach J., Sollich P., Sengupta S., Phys. Rev. Lett., 2020, 124, No. 2, 025503, doi $10.1103 /$ PhysRevLett.124.025503

16. Nath P., Ganguly S., Horbach J., Sollich P., Karmakar S., Sengupta S., PNAS, 2018, 115, No. 19, E4322-E4329, doi $10.1073 /$ pnas.1800837115.

17. Nikoubashman A., Kahl G., Likos C.N., Phys. Rev. Lett., 2011, 107, No. 6, 068302, doi $10.1103 /$ PhysRevLett.107.068302

18. Nikoubashman A., Likos C.N., Kahl G., Soft Matter, 2013, 9, No. 9, 2603-2613, doi 10.1039/C2SM26727F

19. Plimpton S., J. Comput. Phys., 1995, 117, No. 1, 1-19, doi:10.1006/jcph.1995.1039

20. Frenkel D., Smit B., Understanding Molecular Simulation: From Algorithms to Applications, Vol. 1, Academic Press, San Diego, 2001.

21. Soddemann T., Dünweg B., Kremer K., Phys. Rev. E, 2003, 68, No. 4, 046702, doi 10.1103/PhysRevE.68.046702.

22. Stukowski A., Modell. Simul. Mater. Sci. Eng., 2009, 18, No. 1, 015012, doi 10.1088/0965-0393/18/1/015012

23. Lees A., Edwards S., J. Phys. C: Solid State Phys., 1972, 5, No. 15, 1921, doi $10.1088 / 0022-3719 / 5 / 15 / 006$

24. Irving J., Kirkwood J.G., J. Chem. Phys., 1950, 18, No. 6, 817-829, doi:10.1063/1.1747782.

25. Larson R.G., The Structure and Rheology of Complex Fluids, Oxford University Press, Oxford, UK, 1999.

26. Bonn D., Denn M.M., Berthier L., Divoux T., Manneville S., Rev. Mod. Phys., 2017, 89, No. 3, 035005, doi $10.1103 /$ RevModPhys.89.035005.

27. Varnik F., Bocquet L., Barrat J.L., J. Chem. Phys., 2004, 120, No. 6, 2788-2801, doi: 10.1063/1.1636451.

28. Shrivastav G.P., Chaudhuri P., Horbach J., J. Rheol., 2016, 60, No. 5, 835-847, doi:10.1122/1.4959967.

29. Chaudhuri P., Berthier L., Bocquet L., Phys. Rev. E, 2012, 85, No. 2, 021503, doi 10.1103/PhysRevE.85.021503

30. Rottler J., Robbins M.O., Phys. Rev. Lett., 2005, 95, No. 22, 225504, doi:10.1103/PhysRevLett.95.225504

31. Eyring H., J. Chem. Phys., 1936, 4, No. 4, 283-291, doi $10.1063 / 1.1749836$

32. Mladek B.M., Charbonneau P., Frenkel D., Phys. Rev. Lett., 2007, 99, No. 23, 235702, doi $10.1103 /$ PhysRevLett.99.235702 


\section{Про викид напруження в кластерних кристалах під дією зсуву}

\section{Г.П. Шрівастав, Г. Каль}

Інститут теоретичної фізики і центр обчислювального матеріалознавства (CMS), TU Відень, A-1040

Відень, Австрія

Використовуючи симуляції методом нерівноважної молекулярної динаміки, ми досліджуємо поведінку податливості модельного кластерного кристалу, сформованого ультрам'якими частинками під дією зсуву. Ми досліджуємо еволюцію напруження як функцію деформації для різних швидкостей зсуву, $\dot{\gamma}$, і температур. Відношення напруження-деформація показує чіткий максимум в точці податливості; висота цього максимума, $\sigma_{\mathrm{p}}$, зростає за степеневим законом зі збільшенням області зсуву і прямує до насичення до кінцевого значення, коли гранична швидкість зсуву прямує до нуля (принаймні в межах розглянутого діапазону температур). Виявилося, що цю поведінку можна отримати з допомогою моделі Гершеля-Балклі, яка при заданій температурі дозволяє зробити передбачення для статичного податливого напруження

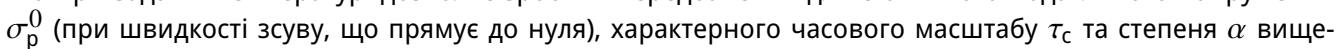
згаданого степенового закону $\sigma_{\mathrm{p}}$ при високих швидкостях зсуву. До того ж, при різних температурах, $\sigma_{\mathrm{p}}$ можуть бути промасштабовані як функції $\dot{\gamma}$ в єдину криву при масштабуванні відповідними значеннями $\tau_{\mathrm{c}}$ і $\sigma_{\mathrm{p}}^{0}$. Крім того, при заданій швидкості зсуву $\sigma_{\mathrm{p}}$ показує логарифмічну залежність від температури. Знову, криві $\sigma_{\mathrm{p}}-T$ для різних швидкостей зсуву можуть бути промасштабовані в єдину логарифмічну криву при масштабуванні відповідними підгоночними параметрами.

Ключові слова: реологія, кластерний кристал, податливість, молекулярна динаміка, перенапруження 


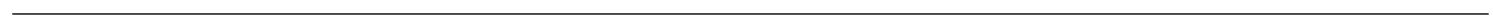

